\title{
TOPOLOGY OF CERTAIN SUBMANIFOLDS IN THE EUCLIDEAN SPHERE
}

\author{
Y. L. XIN
}

ABSTRACT. Using the nonexistence theorem for stable harmonic maps, we study the fundamental group of certain submanifolds in the Euclidean sphere.

1. Introduction. In [3] R. Schoen and S. T. Yau made the first attempt to study the geometry of manifolds by using harmonic maps. They proved that if $M$ is a complete noncompact stable immersed hypersurface in a manifold of nonnegative curvature and $D$ is a compact domain in $M$ with smooth simply connected boundary, then there is no nontrivial homomorphism from $\pi_{1}(D)$ into the fundamental group of a compact manifold with nonpositive curvature.

In this paper we consider certain submanifolds in the Euclidean sphere. First of all we generalize the nonexistence theorem in our previous paper [5] as follows:

Let $M$ be a compact $n$-dimensional immersed submanifold with second fundamental form $B$ and mean curvature $H$ in the Euclidean sphere. When $n>2+\tilde{B}$ there is no nonconstant stable harmonic map from $M$ to any Riemannian manifold $N$, where

$$
\tilde{B}=\left(\sum_{i, j=1}^{n}\left(2\left\langle B_{e_{k}, e_{i}} B_{e_{k}, e_{j}}\right\rangle-\left\langle H, B_{e_{i}, e_{j}}\right\rangle\right)^{2}\right)^{1 / 2} .
$$

According to the J. Simons' theorem [4], when $M$ as above is minimal, it cannot be stable.

Using the above nonexistence theorem and Eells-Sampson's theorem [1], we find a topological restriction similar to that in Schoen-Yau's theorem. The result is the following:

Let $M$ be a compact $n$-dimensional submanifold with second fundamental form $B$ and mean curvature $H$ in the Euclidean sphere. When $n>2+\tilde{B}$ there is no nontrivial homomorphism from the fundamental group $\pi_{1}(M)$ into the fundamental group of a compact manifold with nonpositive curvature.

2. Preliminary notation. We refer the basic notion about harmonic maps to the paper [2]. Our purpose in this section is to sketch the immersed submanifolds.

Let $M$ be a compact $n$-dimensional Riemannian manifold in $\bar{M}$, which is an $m$-dimensional Riemannian manifold. Set $m=n+p$, where $p$ is the codimension

Received by the editors October 7, 1980. The results in this paper have been presented to the Beijing Symposium on Differential Geometry and Partial Differential Equations in the summer of 1980 under the sponsorship of Academia Sinica, Beijing University, Fudan University and the National Academy of Sciences, U.S.A.

1980 Mathematics Subject Classification. Primary 57N15; Secondary 51 H20. 
of $M$ in $\bar{M}$. We shall use the following ranges of indices throughout this paper:

$$
\begin{aligned}
1 & <A, B, C, \ldots<m=n+p, \\
1 & \leqslant i, j, k, l<n, \\
n+1 & \leqslant \alpha, \beta, \gamma, \ldots<n+p .
\end{aligned}
$$

Let $T M$ and $N M$ denote the tangent bundle and the normal bundle of $M$, respectively, such that for any $x \in M \subset \bar{M}$ we have an orthogonal splitting

$$
T_{x}(\bar{M})=T_{x}(M) \oplus N_{x}(M) \text {. }
$$

With respect to this splitting we decompose any vector $X \in T_{x}(\bar{M})$ as $X=(X)^{T} \oplus$ $(X)^{N}$. M inherits the Riemannian connection from one $\bar{\nabla}$ of $\bar{M}$ as follows: let $\tilde{X}$ and $\tilde{Y}$ be vector fields on $M$. Then for $X=\tilde{X}(x)$

$$
\nabla_{x} \tilde{Y}=\left(\bar{\nabla}_{x} \tilde{Y}\right)^{T}
$$

which is the unique Riemannian connection induced by the metric inherited from $\bar{M}$.

The second fundamental form of $M$ in $\bar{M}$ is a section of $\operatorname{Hom}(T M \otimes T M, N M)$, defined as follows: for any $X, Y \in T_{x} M$

$$
B_{X, Y}=\left(\bar{\nabla}_{x} \tilde{Y}\right)^{N}
$$

where $\tilde{Y}$ is an extension of $Y$ to a local tangent vector field on $M$. At each point $x \in M, B_{x}$ represents a symmetric bilinear map of $T_{x} M$ into $N_{x} M$. Thus we can define

$$
H_{x}=\operatorname{trace}\left(B_{x}\right)
$$

for each $x \in M . H$ is called a mean curvature vector field.

Sometimes it is convenient to consider $B$ in adjoint form. For $\nu \in N_{x} M$ we define $A^{\nu}: T_{x} M \rightarrow T_{x} M$ with

$$
A^{\nu}(X)=-\left(\bar{\nabla}_{x} \tilde{\nu}\right)^{T}
$$

for $X \in T_{x} M$, where $\tilde{\nu}$ is any local extension of $\nu$ to a normal vector field. It is easy to check the relation

$$
\left\langle A^{\nu}(X), Y\right\rangle=\left\langle B_{X, Y}, \nu\right\rangle .
$$

We define the squared length of $B$ at each $x \in M$ in the usual way as

$$
\|B\|^{2}=\sum_{i, j=1}^{n}\left\|B_{e, e, j}\right\|^{2}
$$

where $\left\{e_{1}, \ldots, e_{n}\right\}$ is a local orthonormal basis of $M$.

Let $R$ and $\bar{R}$ be the curvature tensors of $M$ and $\bar{M}$, respectively. For any $X, Y, Z$, $W \in T_{x} M$ we have Gauss' formula for submanifolds:

$$
\left\langle R_{X, Y} Z, W\right\rangle=\left\langle\bar{R}_{X, Y} Z, W\right\rangle-\left\langle B_{X, W}, B_{Y, Z}\right\rangle+\left\langle B_{X, Z}, B_{Y, W}\right\rangle,
$$

which we shall have occasion to use below. We adopt the sign convention of [2] about the curvature.

Let us consider a submanifold in the Euclidean sphere $M \subset S^{m} \subset \mathbf{R}^{m+1}$. Let $\theta$ denote the $(n+1)$-dimensional vector space of vector fields on $S^{m}$ by 


$$
\theta=\left\{\left.\operatorname{grad} f\right|_{S^{m}}: f \text { is linear on } \mathbf{R}^{m+1}\right\}
$$

For any $V \in \theta$ there is a unique decomposition $\left.V\right|_{M}=V^{T}+V^{N}$. We denote $\theta^{T}=\left\{V^{T}: V \in \theta\right\}$.

It is easy to check the following relations:

$$
\nabla_{X} V^{N}=-B_{X, V^{T}}
$$

and

$$
\nabla_{X} V^{T}=A^{V^{N}}(X)-f X
$$

3. The proof of the results. Let $M \subset S^{m}$ be a compact immersed submanifold. We consider any harmonic map $\phi: M \rightarrow \bar{M}$, where the image manifold is any Riemannian manifold. By means of this map $\phi$ we obtain an induced vector bundle $\phi^{-1} T M$ over $M$ which inherits a Riemannian connection $\tilde{\nabla}$ from the canonical connection in $\bar{M}$. Choose a local orthonormal basis $\left\{e_{i}\right\}$, such that $\left(\nabla_{e_{i}} e_{j}\right)_{x}=0$ at a given point $x \in M$. For $V^{T} \in \theta^{T}$, taking the cross section $\phi_{*} V^{T}$, we have the following lemmas:

LEMMA 3.1.

$$
\begin{aligned}
\tilde{\nabla}_{e_{i}} \phi_{*}\left(\nabla_{e_{i}} V^{T}\right)= & \left\langle\left(\nabla_{e_{i}} A\right)^{V^{N}}\left(e_{i}\right), e_{j}\right\rangle \phi_{*} e_{j}-\left\langle B_{e_{i}, e_{j}}, B_{e_{i}, V^{T}}\right\rangle \phi_{*} e_{j} \\
& +\left\langle B_{e_{i}, e_{j}}, V^{N}\right\rangle \tilde{\nabla}_{e_{i}} \phi_{*} e_{j}-\phi_{*} V^{T} .
\end{aligned}
$$

Proof. Using (2.5), (2.8) and (2.9), we have

$$
\begin{aligned}
& \tilde{\nabla}_{e_{i}} \phi_{*}\left(\nabla_{e_{i}} V^{T}\right)=\tilde{\nabla}_{e_{i} \phi_{*}}\left(A^{V^{N}}\left(e_{i}\right)-f e_{i}\right) \\
& =\tilde{\nabla}_{e_{i}} \phi_{*}\left\langle A^{V^{N}}\left(e_{i}\right), e_{j}\right\rangle e_{j}-\left\langle V^{T}, e_{i}\right\rangle \phi_{*} e_{i} \\
& =\tilde{\nabla}_{e_{i}}\left\langle A^{V^{N}}\left(e_{i}\right), e_{j}\right\rangle \phi_{*} e_{j}-\phi_{*} V^{T} \\
& =\left\langle\nabla_{e_{i}} A^{V^{N}}\left(e_{i}\right), e_{j}\right\rangle \phi_{*} e_{j}+\left\langle A^{V^{N}}\left(e_{i}\right), e_{j}\right\rangle \tilde{\nabla}_{e_{i}} \phi_{*} e_{j}-\phi_{*} V^{T} \\
& =\left\langle\left(\nabla_{e_{i}} A\right)^{V^{N}}\left(e_{i}\right), e_{j}\right\rangle \phi_{*} e_{j}+\left\langle A^{\nabla_{i} V^{N}}\left(e_{i}\right), e_{j}\right\rangle \phi_{*} e_{j} \\
& +\left\langle B_{e_{i}, e_{j}}, V^{N}\right\rangle \tilde{\nabla}_{e_{i}} \phi_{*} e_{j}-\phi_{*} V^{T} \\
& =\left\langle\left(\nabla_{e_{i}} A\right)^{V^{N}}\left(e_{i}\right), e_{j}\right\rangle \phi_{*} e_{j}-\left\langle B_{e_{i}, e_{j}}, B_{e_{i}, V^{T}}\right\rangle \phi_{*} e_{j} \\
& +\left\langle B_{e_{i}, e_{j}}, V^{N}\right\rangle \tilde{\nabla}_{e_{i} \phi_{*}} e_{j}-\phi_{*} V^{T} \text {. Q.E.D. }
\end{aligned}
$$

LEMMA 3.2.

$$
\varphi_{*}\left(\nabla^{*} \nabla V^{T}\right)=\left\langle\left(\nabla_{e_{i}} A\right)^{V^{N}}\left(e_{i}\right), e_{j}\right\rangle \phi_{*} e_{j}-\left\langle B_{e_{i}, V^{T}}, B_{e_{i}, e_{j}}\right\rangle \phi_{*} e_{j}-\phi_{*} V^{T}
$$

Proof. Using (2.5), (2.8) and (2.9), we obtain

$$
\begin{aligned}
\phi_{*}\left(\nabla^{*} \nabla V^{T}\right) & =\phi_{*}\left(\nabla_{e_{i}} \nabla_{e_{i}} V^{T}\right)=\phi_{*} \nabla_{e_{i}}\left(A^{V^{N}}\left(e_{i}\right)-f e_{i}\right) \\
& =\phi_{*}\left(\left(\nabla_{e_{i}} A\right)^{V^{N}}\left(e_{i}\right)+A^{\nabla_{i} V^{N}}\left(e_{i}\right)-V^{T}\right) \\
& =\phi_{*}\left(\left\langle\left(\nabla_{e_{i}} A\right)^{V^{N}}\left(e_{i}\right), e_{j}\right\rangle e_{j}-\left\langle B_{e_{i}, V^{T}}, B_{e_{i}, e_{j}}\right\rangle e_{j}-V^{T}\right) \\
& =\left\langle\left(\nabla_{e_{i}} A\right)^{V^{N}}\left(e_{i}\right), e_{j}\right\rangle \phi_{*} e_{j}-\left\langle B_{e_{i}, V^{T}}, B_{e_{i}, e_{j}}\right\rangle \phi_{*} e_{j}-\phi_{*} V^{T} .
\end{aligned}
$$


646

Y. L. IN

LEMMA 3.3.

$$
\begin{aligned}
-\tilde{\nabla} * \tilde{\nabla} \phi_{*} V^{T}= & R^{\bar{M}}\left(\phi_{*} e_{i}, \phi_{*} V^{T}\right) \phi_{*} e_{i}+(2-n) \phi_{*} V^{T} \\
& -\left\langle\left(\nabla_{e_{i}} A\right)^{V^{N}}\left(e_{i}\right), e_{j}\right\rangle \phi_{*} e_{j}-\left\langle H, B_{V^{T}, e_{j}}\right\rangle \phi_{*} e_{j} \\
& -2\left\langle B_{e_{i}, e,}, V^{N}\right\rangle \tilde{\nabla}_{e_{i}} \phi_{*} e_{j}+2\left\langle B_{e_{i}, e}, B_{e_{i}, V^{T}}\right\rangle \phi_{*} e_{j}
\end{aligned}
$$

Proof. In our case $M \subset S^{m}$ by using Gauss formula (2.7), we have

$$
\begin{aligned}
\left\langle R_{X Y} Z, W\right\rangle= & \langle X, Z\rangle\langle Y, W\rangle-\langle X, W\rangle\langle Y, Z\rangle \\
& -\left\langle B_{X, W}, B_{Y, Z}\right\rangle+\left\langle B_{X, Z}, B_{Y, W}\right\rangle .
\end{aligned}
$$

Hence

$$
\begin{aligned}
\langle\operatorname{Ric} Y, W\rangle & =\left\langle R_{e_{i} Y} e_{i}, W\right\rangle \\
& =(n-1)\langle Y, W\rangle-\left\langle B_{e_{i}, W}, B_{e_{i}, Y}\right\rangle+\left\langle H, B_{Y, W}\right\rangle .
\end{aligned}
$$

Namely

$$
\text { Rid } Y=(n-1) Y-\left\langle B_{e_{i}, Y}, B_{e_{i}, e_{j}}\right\rangle e_{j}+\left\langle H, B_{Y, e_{j}}\right\rangle e_{j} \text {. }
$$

By Weitzenböck's formula and (3.4)

$$
\begin{aligned}
& -(\tilde{\nabla} * \tilde{\nabla} d \phi) V^{T}=R^{\bar{M}}\left(\phi_{*} e_{i}, \phi_{*} V^{T}\right) \phi_{*} e_{i}-\phi_{*}\left(\operatorname{Ric} V^{T}\right) \\
& =R^{\bar{M}}\left(\phi_{*} e_{i}, \phi_{*} V^{T}\right) \phi_{*} e_{i}-(n-1) \phi_{*} V^{T} \\
& +\left\langle B_{e_{i}, V^{T}}, B_{e_{i}, e_{j}}\right\rangle \phi_{*} e_{j}-\left\langle H, B_{V^{T}, e_{j}}\right\rangle \phi_{*} e_{j}
\end{aligned}
$$

Thus from (3.1), (3.2) and (3.5), we have

$$
\begin{aligned}
& -\tilde{\nabla} * \tilde{\nabla}_{\phi_{*}} V^{T}=-\tilde{\nabla}_{e_{i}} \tilde{\nabla}_{e_{i}} d \phi\left(V^{T}\right) \\
& =-\tilde{\nabla}_{e_{i}}\left(\left(\tilde{\nabla}_{e_{i}} d \phi\right) V^{T}+d \phi\left(\nabla_{e_{i}} V^{T}\right)\right) \\
& =-\left(\tilde{\nabla}_{e_{i}} \tilde{\nabla}_{e_{i}} d \phi\right) V^{T}-2\left(\tilde{\nabla}_{e_{i}} d \phi\right) \nabla_{e_{i}} V^{T}-d \phi\left(\nabla_{e_{i}} \nabla_{e_{i}} V^{T}\right) \\
& =-\left(\tilde{\nabla}_{e_{i}} \tilde{\nabla}_{e_{i}} d \phi\right) V^{T}-2 \tilde{\nabla}_{e_{i}} \phi_{*}\left(\nabla_{e_{i}} V^{T}\right)+\phi_{*}\left(\nabla_{e_{i}} \nabla_{e_{i}} V^{T}\right) \\
& =R^{\bar{M}}\left(\phi_{*} e_{i}, \phi_{*} V^{T}\right) \phi_{*} e_{i}-(n-1) \phi_{*} \dot{V}^{T} \\
& +\left\langle B_{e_{i}, V^{r}}, B_{e_{i}, e_{j}}\right\rangle \phi_{*} e_{j}-\left\langle H, B_{V^{T}, e_{j}}\right\rangle \phi_{*} e_{j} \\
& -2\left\langle\left(\nabla_{e_{i}} A\right)^{V^{N}}\left(e_{i}\right), e_{j}\right\rangle \phi_{*} e_{j}+2\left\langle B_{e_{i}, e_{j}}, B_{e_{i}, V^{T}}\right\rangle \phi_{*} e_{j} \\
& -2\left\langle B_{e_{i}, e_{j}}, V^{N}\right\rangle \tilde{\nabla}_{e_{i}} \phi_{*} e_{j}+2 \phi_{*} V^{T} \\
& +\left\langle\left(\nabla_{e_{i}} A\right)^{V^{N}}\left(e_{i}\right), e_{j}\right\rangle \phi_{*} e_{j}-\left\langle B_{e_{i}, V^{T}}, B_{e_{i}, e_{j}}\right\rangle \phi_{*} e_{j}-\phi_{*} V^{T} \\
& =R^{\bar{M}}\left(\phi_{*} e_{i}, \phi_{*} V^{T}\right) \phi_{*} e_{i}+(2-n) \phi_{*} V^{T}-\left\langle\left(\nabla_{e_{i}} A\right)^{V^{N}}\left(e_{i}\right), e_{j}\right\rangle \phi_{*} e_{j} \\
& -\left\langle H, B_{V^{T}, e_{j}}\right\rangle \phi_{*} e_{j}-2\left\langle B_{e_{i}, e_{j},}, V^{N}\right\rangle \tilde{\nabla}_{e_{i} \phi_{*} e_{j}}+2\left\langle B_{e_{i, e},}, B_{e_{i}, V^{T}}\right\rangle \phi_{*} e_{j}
\end{aligned}
$$


Using the second variation formula for harmonic maps, we have

$$
\begin{aligned}
i= & I\left(\phi_{*} V^{T}, \phi_{*} V^{T}\right) \\
= & \int_{M}\left\langle-\tilde{\nabla} * \tilde{\nabla} \phi_{*} V^{T}-R^{\bar{M}}\left(\phi_{*} e_{i}, \phi_{*} V^{T}\right) \phi_{*} e_{i}, \phi_{*} V^{T}\right\rangle * 1 \\
= & \int_{M}\left\{(2-n)\left\|\phi_{*} V^{T}\right\|^{2}\right. \\
& \quad-\left[\left\langle\left(\nabla_{e_{i}} A\right)^{V^{N}}\left(e_{i}\right), e_{j}\right\rangle+2\left\langle B_{e_{i}, e_{j}}, B_{e_{i}, V^{T}}\right\rangle-\left\langle H, B_{V^{T}, e_{j}}\right\rangle\right] \\
& \cdot\left\langle\phi_{*} e_{j}, \phi_{*} V^{T}\right\rangle-2\left\langle B_{e_{i}, e_{j}}, V^{N}\right\rangle\left\langle\tilde{\nabla}_{\left.\left.e_{i} \phi_{*} e_{j}, \phi_{*} V^{T}\right\rangle\right\} * 1 .}\right.
\end{aligned}
$$

Now we choose an orthonormal basis $\left\{x, e_{i}, \nu_{\alpha}\right\}$ for $\mathbf{R}^{N+1}$, where $e_{i}$ are (parallel to) tangent vectors to $M$ at the point $x \in M$. This basis determines an orthonormal basis $\left\{X, E_{i}, F_{\alpha}\right\}$ for $\theta$ and a corresponding basis $\left\{X^{T}, E_{i}^{T}, F_{\alpha}^{T}\right\}$ for $\theta^{T}$ such that $X(x)=0, E_{i}(x)=e_{i}$ and $F_{\alpha}(x)=\nu_{\alpha}$, namely $E_{i}^{T}(x)=e_{i}, E_{i}^{N}(x)=0, F_{\alpha}^{T}(x)=0$ and $F_{\alpha}^{N}(x)=\nu_{\alpha}$. Hence

$$
\operatorname{trace} i=(2-n) E(\phi)+\int_{M}\left[2\left\langle B_{e_{k}, e_{j}}, B_{e_{k}, e_{i}}\right\rangle-\left\langle H, B_{e_{i}, e_{j}}\right\rangle\right]\left\langle\phi_{*} e_{i}, \phi_{*} e_{j}\right\rangle * 1
$$

where $E(\phi)$ is the energy integral of the harmonic map $\phi$.

We have the following lemma whose proof is not difficult; we leave it to the readers.

LEMMA 3.4. If $A$ and $B$ are symmetric matrices and $B$ is semipositive definite, then trace $A B<\left(\text { trace } A^{2}\right)^{1 / 2}$ trace $B$.

Therefore (3.7) becomes

$$
\text { trace } i \leqslant(2-n) E(\phi)+\int_{M} \tilde{B}\left\langle\phi_{*} e_{k}, \phi_{*} e_{k}\right\rangle * 1,
$$

where

$$
\tilde{B}=\left(\sum_{i, j=1}^{n}\left(2\left\langle B_{e_{k}, e_{i}}, B_{e_{k}, e_{j}}\right\rangle-\left\langle H, B_{e_{i}, e_{j}}\right\rangle\right)^{2}\right)^{1 / 2} .
$$

Thus we obtain the following:

THEOREM 1. Let $M$ be an n-dimensional compact submanifold with second fundamental form $B$ and mean curvature $H$ in the Euclidean sphere $S^{m}$. When $n>2+\tilde{B}$ there is no nonconstant stable harmonic map from $M$ to any Riemannian manifold, where

$$
\tilde{B}=\left(\sum_{i, j=1}^{n}\left(2\left\langle B_{e_{k}, e_{i}}, B_{e_{k}, e_{j}}\right\rangle-\left\langle H, B_{e_{i}, e_{j}}\right\rangle\right)^{2}\right)^{1 / 2} .
$$

Remark. If $B=O, M$ is a sphere $S^{n}$ with the usual totally geodesic imbedding, this theorem becomes Theorem 3.1 of our previous paper [5].

Using the above Theorem 1 , we obtain a certain topological restriction on $M$. 
TheOREM 2. Let $M$ be an $n$-dimensional compact submanifold with second fundamental form $B$ and mean curvature $H$ in the Euclidean sphere $S^{m}$ and let $\bar{M}$ be $a$ compact Riemannian manifold with nonpositive sectional curvature. If $n>2+\tilde{B}$, then there is no nontrivial homomorphism from the fundamental group $\pi_{1}(M)$ into $\pi_{1}(\bar{M})$, where

$$
\tilde{B}=\left(\sum_{i, j=1}^{n}\left(2\left\langle B_{e_{k}, e_{i}}, B_{e_{k}, e_{j}}\right\rangle-\left\langle H, B_{e_{i}, e_{j}}\right\rangle\right)^{2}\right)^{1 / 2} .
$$

Proof. Let $h: \pi_{1}(M) \rightarrow \pi_{1}(\bar{M})$ be a homomorphism. Since $M$ is compact and $\bar{M}$ is $K(\pi, 1)$, there exists a smooth map $f: M \rightarrow \bar{M}$, such that its induced map $f_{*}$ between the fundamental groups is $h$. By Eells-Sampson's theorem [1] there exists a harmonic map $\phi$ which is homotopic to $f$ and has minimum energy in its homotopy class. It follows both that $\phi_{*}=h$ and that $\phi$ is a stable harmonic map. But Theorem 1 tells us $\phi$ is constant so that $h$ is trivial. Q.E.D.

Acknowledgement. The author is gratefully indebted to Professor C. N. Yang for his encouragement and concern. He would like to thank Professor C. K. Peng for helpful discussions. He is also grateful to the State University of New York at Stony Brook for its hospitality.

\section{REFERENCES}

1. J. Eells and J. H. Sampson, Harmonic mappings of Riemannian manifolds, Amer. J. Math. 86 (1964), 109-160.

2. J. Eells and L. Lemaire, A report on harmonic maps, Bull. London Math. Soc. 10 (1978), 1-68.

3. R. Schoen and S. T. Yau, Harmonic maps and topology of stable hypersurfaces and manifolds with non-negative Ricci curvature, Comment. Math. Helv. 39 (1976), 333-341.

4. J. Simons, Minimal varieties in Riemannian manifolds, Ann. of Math. (2) 88 (1968), 62-105.

5. Y. L. Xin, Some results on harmonic maps, Duke Math. J. 47 (1980), 609-613.

Institute for Theoretical Physics, State University of New York at Stony Brook, Stony BROOK, NEW YORK 11794

Department of Mathematics, Fudan University, Shanghai, People's Republic of China 